LVOV MATHEMATICAL SCHOOL

IN THE PERIOD 1915-45 AS SEEN TODAY

BANACH CENTER PUBLICATIONS, VOLUME 87

INSTITUTE OF MATHEMATICS

POLISH ACADEMY OF SCIENCES

WARSZAWA 2009

\title{
BANACH'S SCHOOL AND TOPOLOGICAL ALGEBRAS
}

\author{
WIESŁAW ŻELAZKO \\ Mathematical Institute, Polish Academy of Sciences \\ Śniadeckich 8, P.O. Box 21, PL-00-956 Warszawa, Poland \\ E-mail: zelazko@impan.pl
}

\begin{abstract}
We present here some evidence of the activity of Banach Lwów School of functional analysis in the field of topological algebras. We shall list several results connected with such names as Stanisław Mazur (1905-1981), Maks (Meier) Eidelheit (1910-1943), Stefan Banach (1892-1945) and Andrzej Turowicz (1904-1989) showing that if the war had not interrupted this activity we could expect more interesting results in this direction.
\end{abstract}

1. The Mazur-Gelfand theorem. This is perhaps the most spectacular and best known result of the Lwów School in the field of topological algebras. It has many further proofs and many generalizations. It says that a real normed division algebra (Mazur does not assume here its completeness, but assumes the submultiplicativity of the norm) is topologically isomorphic either with the field of complex numbers, or with the field of real numbers or with the division algebra of real quaternions. Mazur published this result in [12] omitting its proof (present in the original manuscript), since the paper was too long for publication in Comptes Rendus. A similar result (for complex algebras and under assumption of completeness) was obtained later, perhaps independently, by I. M. Gelfand [7] who based on this result his theory of ideals in commutative complex Banach algebras. In the paper [6], announcing some results of [7], there is no mention about Mazur's result, but in [7] Gelfand says that this result is due to Mazur. Gelfand's proof is simpler and essentially uses the fact that the algebra is complex. It seems that the reason that Mazur considered a more complicated case of a real algebra is that the Banach School worked only with real Banach spaces.

Thanks to the kindness of Professor Pierre Mazet (see [11]), who provided me with the original text of Mazur which was omitted in the paper [12], I quote literally this proof below (this is the reason that it is numbered 1, while other results in this paper are numbered with two digits; it is different from the text published in my book [19], which

2000 Mathematics Subject Classification: Primary 01A72; Secondary 46-03.

The paper is in final form and no version of it will be published elsewhere. 
I have obtained from Stanisław Mazur). Stanisław Mazur asked the author whether his original proof should be published, my opinion was that it should not, since it was already published in my book, and he agreed. The term "space of type $\left(B^{*}\right)$ " means here a not necessarily complete normed space.

THÉORÈme 1. Chaque domaine de rationalité de type $\left(B^{*}\right)$ est isomorphe au domaine de rationalité des nombres réels, des nombres complexes ou des quaternions.

On voit facilement en s'appuyant sur le théorème de G. Frobenius et la remarque (b) qu'il suffit d'établir la non-existence d'une norme dans le domaine de rationalité $R$ de toutes les fonctions rationnelles à coefficients réels, ce qu'on établit par le raisonnement suivant: Supposons qu'il existe une norme dans $R$. Soient $x$ et $y$ des nombres réels, $z=x+i y, t$ une variable réelle, $\varphi(X)$ une fonctionnelle linéaire dans $R$; en désignant par $\mathcal{R}(w)$ la partie réelle d'un nombre complexe $w$, posons

$$
f(x, y)=\varphi\left(\mathcal{R}\left(\frac{1}{z-t}\right)\right) .
$$

La fonction $f(x, y)$ est harmonique dans le plan entier, y compris le point à l'infini; en effet, étant donnés les nombres réels $x_{0}, y_{0}$ et $z_{0}=x_{0}+i y_{0}$ on a

$$
f(x, y)=\sum_{n=1}^{\infty} \varphi\left(\left(\frac{\left(z-z_{0}\right)^{n}}{\left(z_{0}-t\right)\left(t-z_{0}\right)^{n}}\right)\right)
$$

dans un certain voisinage du point $\left(x_{0}, y_{0}\right)$, la série étant uniformément convergente et ses termes étant des fonctions harmoniques; de même dans un certain voisinage du point à l'infini la fonction admet une représentation analogue

$$
f(x, y)=\sum_{n=1}^{\infty} \varphi\left(\mathcal{R}\left(\frac{t^{n}}{z^{n+1}}\right)\right) .
$$

La fonction $f(x, y)$ serait donc constante. Or cela est impossible puisque $\left(x_{1}, y_{1}\right),\left(x_{2}, y_{2}\right)$ étant deux points différents et $z_{k}=x_{k}+y_{k}$, il existe, d'après un théorème fondamental une fonctionnelle linéaire $\varphi(X)$ dans $R$ telle que

$$
\varphi\left(\mathcal{R}\left(\frac{1}{z_{1}-t}\right)\right) \neq \varphi\left(\mathcal{R}\left(\frac{1}{z_{2}-t}\right)\right) .
$$

Mazur gave the following corollary to the above result: Let $T$ be a linear operator on a normed space $X$. Then there are real scalars $\alpha, \beta$ such that either the equation

$$
T^{2} x+\alpha T x+\beta x=0
$$

has a non-zero solution in $X$, or the equation

$$
T^{2} x+\alpha T x+\beta x=y
$$

has no solution for some $y$ in $X$.

The paper [11] contains also two other (less known) results, their formulation here is not identical with their original formulation. 
THEOREM 1.2. Let $A$ be a normed algebra with norm satisfying

$$
\|x y\|=\|x\|\|y\|, x, y \in A .
$$

Then the conclusion of Theorem 1.1 holds true.

Later a more general result was obtained by Kaplansky ([10], Theorem 3.1), who proved that a normed algebra without topological divisors of zero is a division algebra.

THEOREM 1.3. If $A$ is a Banach algebra with all elements algebraic, then there is an integer $n$ such that all elements of $A$ are algebraic of order at most $n$.

2. The uniqueness of norm topology. Eidelheit obtained the first result concerning the uniqueness of topology for a semisimple Banach algebra in the particular case of the algebra $L(X)$ of all bounded operators on a Banach space $X$. As Eidelheit writes in the introduction to the paper [4], the problem was suggested to him by S. Mazur; he also says that this paper is connected with Mazur's research on general linear algebras and quotes here the paper [12].

His result reads as follows (as in other publications of the Banach School all Banach spaces and algebras considered are real).

THEOREM 2.1. Let $X$ be a Banach space. If the algebra $L(X)$ is complete under a submultiplicative norm, then this norm is equivalent with the operator norm.

Nowadays this result is covered by the much more general famous result of Barry Johnson [9] about uniqueness of the norm topology in semisimple Banach algebras (such a result for commutative algebras was already given by Gelfand in [7]). However Johnson's and Gelfand's results are formulated for complex Banach algebras, so some reasoning is needed to deduce from them the corresponding results for real algebras.

Other results of the paper [4] are also of substantial interest.

THEOREM 2.2. Let $X_{1}$ and $X_{2}$ be Banach spaces and suppose that there is an (algebraic) isomorphism $\Phi$ between the algebras $L\left(X_{1}\right)$ and $L\left(X_{2}\right)$. Then the spaces $X_{1}$ and $X_{2}$ are topologically isomorphic. Moreover, the isomorphism $\Phi$ is given by the formula

$$
\Phi(T)=S T S^{-1},
$$

where $S$ is a one-to-one continuous linear operator from $X_{1}$ onto $X_{2}$.

COROLlary. Every automorphism of $L(X)$ is inner.

The third result of the paper [4] concerns multiplicative maps only.

TheOrem 2.3. Let $X_{1}$ and $X_{2}$ be Banach spaces with $\operatorname{dim} X_{1} \geq 2$ and let $\Phi$ be a one-toone continuous multiplicative map of $L\left(X_{1}\right)$ onto $L\left(X_{2}\right)$. Then $\Phi$ is linear and is of the form (1).

Theorems 2.1 and 2.2 are reproduced in Palmer's book [13], pp. 105-106.

Eidelheit wrote also another paper [5]; it concerns uniform algebras. Answering a question posed by Mazur, he proved that any unital closed subalgebra of the algebra $C(T)$, where $T$ is a compact metric space, is isometrically isomorphic with some algebra 
$C\left(T_{1}\right)$, where $T_{1}$ is also compact and metric. Similarly as in the previous paper, the result is obtained for real algebras.

3. The continuity of an inverse. A result found in the notes of Banach, and posthumously published by Hartman [2] says that if $G$ is a group which is a complete metric space, and the multiplication on $G$ is separately continuous, then the operation $x \mapsto x^{-1}$ is also continuous. Banach deduced from this result the following result concerning algebras of type $F$, i.e. complete metric topological algebras.

TheOREM 3.1. Let $A$ be a unital algebra of type $F$. Then the operation of taking inverse is continuous on the set (group) $G(A)$ of all invertible elements of $A$ if and only if $G(A)$ is a $G_{\delta}$-set.

The result on groups mentioned above was published in 1936 by Montgomery [13] under the separability condition and was later used by Arens [1] for obtaining the Mazur-Gelfand type theorem for locally convex separable $F$-algebras ( $B_{0}$-algebras). In the same paper Arens obtained also the Theorem 3.1 for separable algebras, using the Montgomery's result. In fact, the proofs of the results in [2] work only under the separability condition (Hartman has overlooked this fact). Later the author obtained [17], the Montgomery's result without the separability condition, obtaining in this way the Mazur-Gelfand theorem for non-separable algebras of type $B_{0}$. The result was proved for commutative groups; it was sufficient for obtaining the Mazur-Gelfand theorem for $B_{0}$-algebras. However, Gleason in his review [8] of [17] indicated how one can modify the proof in order to obtain the result also in the non-commutative case. The resulting general version of Arens-Banach theorem (above Theorem 3.1) was formulated in ([18], Theorem 7.4).

A corollary stated in [2] says that if $A$ is a division algebra of type $F$, then the operation of taking inverse is continuous there. That seems to be a necessary step for proving (if true) the Mazur-Gelfand theorem for $F$-algebras and perhaps Mazur and others tried to prove this result. The problem whether it is true is still open.

4. A generalization of Weierstrass theorem. Andrzej Turowicz (having a position in Kraków) worked temporarily in Lwów since 1937 and after obtaining the results described in Section 1 Mazur invited Turowicz for further collaboration in Banach algebras. The collaboration started in the fall of 1938 and by April 1939 they obtained several (over 20) results, one of them known as the Stone-Weierstrass theorem. A suitable paper was written by Turowicz and handed to Mazur for publication, but it was never published. The author has heard a rumour that Mazur and Turowicz had the Stone-Weierstrass theorem before Stone, and asked Turowicz about it. In response he obtained a long letter, asking not to publish it before Turowicz's death. The letter was published in [16]. Here follow (in translation) some excerpts from it: “... The discussion was useless in view of Mazur's habit of not publishing the results immediately.... In 1940 I learned that the next volume of Studia Math. will appear, the only one published during the war. I asked Mazur whether we could include our paper there, but he answered that the volume was already closed and there was no more space. In 1941, after taking up Lwów by Germans, 
I left for Kraków and lost contact with Mazur. At the beginning of the fifties Mazur told Ważewski that he had found our manuscript, and Ważewski repeated it to me. Then I wrote to Mazur, suggesting that some of our results could still be published, and asked him to send me the manuscript, so that I could see which results are not yet published and prepare a suitable publication. Of course, I knew that the priority with respect to the generalization of the Weierstrass theorem belongs now to Stone. Mazur never answered my letter; I was not surprised knowing that he had a habit of not answering letters.

I regret not having published the "Weierstrass-Stone" theorem, but what can be done, it's lost. I know that after the war, Orlicz, when collaborating with Mazur, made notes and published a joint paper without asking Mazur for permission. Unfortunately I did not know that I should have done the same."

Turowicz wrote also in his letter that he does not remember the other results of the paper, but, as far as he remembers, the proof of the Weierstrass-Stone theorem was not more difficult than the other proofs. The manuscript has not been found after Mazur's death.

The author has heard the following story (not included in the letter of Turowicz): When Mazur learned about Stone's generalization of Weierstrass theorem, he approached Turowicz and said "we do not have to publish our paper since one of our essential results has already appeared, but the author did not obtain all our results". The author is not sure whether this story is true.

5. Continuous multiplicative functionals. The result described below was suggested to Turowicz by Banach and Eidelheit, evidently during Turowicz's stay in Lwów in 193741, while it was published after the war in the paper [15]. It is related to the Theorem 2.3 of Eidelheit described above. Let $E$ be a compact metric space and consider the uniform algebra $C(E)$ of all continuous real-valued functions. Denote by $\Phi$ the set of all continuous multiplicative functionals $F$ on $C(E)$, i.e. $f(x y)=f(x) f(y)$ for all $x, y \in C(E)$ The following is the main result of the paper [15].

THEOREM 5.1. We have $F \in \Phi$ if and only if the following holds true.

$1^{\circ}$. There is an at most countable non-void subset $Z \subset E$, its points are denoted by $t_{1}, t_{2}, \ldots$;

$2^{\circ}$. There is a sequence $\left(\alpha_{i}\right)$ of positive numbers with $\sum_{i} \alpha_{i}<\infty$;

$3^{\circ}$. There is a sequence $\left(\beta_{i}\right), \beta_{i}=1$ or 2 ;

such that

$$
\begin{aligned}
F(x)= & \prod_{i=1}^{\infty}\left|x\left(t_{i}\right)\right|^{\alpha_{i}} \operatorname{sgn}^{\beta_{1}} x\left(t_{1}\right) \operatorname{sgn}^{\beta_{2}} x\left(t_{2}\right) \\
& \times \prod_{1}^{\infty}\left[\operatorname{sgn} x\left(t_{2 i+1}\right) \operatorname{sgn} x\left(t_{2 i+2}\right)\right]^{\beta_{i+2}} .
\end{aligned}
$$

In the case when $Z$ is finite, the above products are finite. 
We must say that the questions connected with (only) multiplicative operators (as in Theorem 2.3) or functionals did not receive much attention in the past.

It has to be noted that an analogous result for complex algebras is not known.

The above shows that in the early stage of development of topological (in particular Banach) algebras the Banach school obtained several results, some of them quite importrant and some, as far, not present in the mainstream of the theory. The good chances of further development of this theory were interrupted for many years by the war (in the case of Banach's school, by invasion of Germans on the Soviet Union in June 1941, since the Lwów University continued its work under Soviets, and even Banach became the dean of Department of Mathematics). Eidelheit perished in 1943 at the age of 33. Turowicz entered a monastery immediately after the war, and while he continued working as a mathematician, he changed the field of his interest to numerical methods. Banach was offered a chair at the Jagiellonian University (University of Kraków) but he died of cancer in 1945 before taking it. It seems that after the war Mazur stopped working in algebras (it is unclear when he considered the problem of continuity of characters in $B_{0}$-algebras, known as Michael's, or Michael-Mazur problem. He only reduced the problem to some algebra of analytic functions in countably many variables, obtained also much later by Dixon and Esterle ([3], Proposition 2.1) in a more general context of non-commutative algebras; these results of Mazur are known only from oral communications and were never published).

One can only repeat after Turowicz: "what can be done, it's lost".

\section{References}

[1] R. F. Arens, Linear topological division algebras, Bull. Amer. Math. Soc. 53 (1947), 623630 .

[2] S. Banach, Remarques sur les groupes et corps métriques, Studia Math. 10 (1948), 178-181.

[3] P. G. Dixon and J. R. Esterle, Michael's problem and the Poincaré-Fatou-Bieberbach phenomenon, Bull. Amer. Math. Soc. (New Series) 15 (1986), 127-187.

[4] M. Eidelheit, On isomorphisms of rings of linear operators, Studia Math. 9 (1940), 97-105.

[5] - Concerning rings of continuous functions, Ann. of Math. 41 (1940), 393-393.

[6] I. Gelfand, On normed rings, Dokl. A. N. SSSR 23 (1939), 430-432.

[7] —, Normierte Ringe, Mat. Sb. 9 (1941), 1-23.

[8] A. Gleason, Math. Rev. 23 \#A3198.

[9] B. E. Johnson, The uniqueness of the (complete) norm topology, Bull. Amer. Math. Soc. 73 (1967), 537-539.

[10] I. Kaplansky, Normed algebras, Duke Math. J. 16 (1949), 399-418.

[11] P. Mazet, Le preuve originale de S. Mazur pour son théorème sur les algèbres normées, Gaz. Math. Soc. Math. France 111 (2007), 5-11.

[12] S. Mazur, Sur les anneaux linéaires, C. R. Acad. Sci. Paris 207 (1938), 1025-1028. 
[13] D. Montgomery, Continuity in topological groups, Bull. Amer. Math. Soc. 42 (1936), 279282.

[14] T. W. Palmer, Banach Algebras and The General Theory of *-Algebras, Cambridge University Press, 1994.

[15] A. Turowicz, Sur les fonctionnelles continues et multiplicatives, Ann. Soc. Pol. Math. 20 (1947), 135-166.

[16] -, Concerning the proof of the Weierstrass-Stone theorem (in Polish), Wiad. Mat. 31 (1995), 149-150.

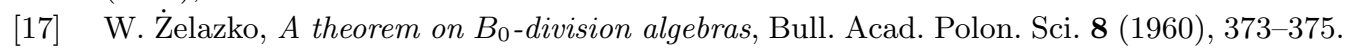

[18] —, Metric Generalizations of Banach Algebras, Dissert. Math. (Rozprawy Mat.) 47, 1965.

[19] —, Banach Algebras, PWN-Elsevier, 1973. 
